\title{
Rotating Capacitor and a Transient Electric Network
}

\author{
Haiduke Sarafian $^{1}$ and Nenette Sarafian ${ }^{2}$ \\ ${ }^{1}$ The Pennsylvania State University \\ University College \\ York, PA 17403 \\ has2@psu.edu \\ ${ }^{2}$ Penn State Shock Trauma Center \\ The Milton S. Hershey Medical Center \\ Hershey, PA 17033 \\ nsarafian@hmc.psu.edu
}

\begin{abstract}
The authors designed a rotating parallel-plate capacitor; one of the plates is assumed to turn about the common vertical axis through the centers of the square plates. We insert this capacitor into a series with a resistor, forming a RC circuit. We analyze the characteristics of charging and discharging scenarios on two different parallel tracks. On the first track we drive the circuit with a DC power supply. On the second track, we drive the circuit with an AC source.

The analyses of the circuits encounter non-linear differential equations. We format the underlying equations into their generic forms. We then apply Mathematica 1, NDSolve to solve them. This work is an example showing how with the help of Mathematica one is able to augment the scope of the traditional studies.
\end{abstract}

Keywords: Mathematica, Electric Network, Geometry.

\section{Introduction and Motivation}

It is a far-fetched concept to think about a transient electrical circuit and incorporate its characteristics to a discrete and abstract geometrical problem. The authors have even taken the initiative one step further, relating these two basic concepts to the kinematics of mechanics. In other words, this article shows how these three discrete concepts are brought together and molded into one coherent and unique project. To accomplish this, one needs to think creatively, Mathematica is the tool of choice helping to explore the possibilities. This article including the Introduction is composed of eight sections. In Section 2, we apply Mathematica to evaluate the overlapping area of the two rotating squares about their common vertical axis. In section 3 we incorporate the rotational kinematics and consider two different scenarios: 1) a symmetrical, uniform rotation; and 2) an asymmetrical, accelerated rotation.

In Section 4-7, we view the overlapping squares as being two parallel metallic plates that are separated by a gap forming a parallel-plate capacitor. Since the 
area of the overlapping plates evaluates the capacitance of the capacitor, the rotating plates make the capacitor a variable one. Technical literature particularly Mathematica-based articles and reports lack one such view. It is the ultimate objective of this project to analyze the response of the electrical circuits to the kinematics of the rotating plates.

Specifically, in this article, we address the modifications of the basic responses of the electrical circuits composed of a resistor connected in a series with our designed, time-dependent capacitor. In particular, we analyze the characteristics of the RC circuits driven with DC as well as AC sources. In conjunction with our analysis, in section 8 we close the article suggesting a few related research flavored circuit analysis projects.

\section{Analysis}

Figure 1 shows two identical overlapping squares. The bottom square designated with non-prime vertices is fastened to the xy coordinate system. The top square, designated with prime vertices is rotated counter clockwise about the common vertical axis through the common origin $\mathrm{O}$ by an angle $\theta$. The squares have the side length of $L$ and the rotation angle $\theta$ is the angle between the semi diagonals $O P_{1}$ and $O P_{1}^{\prime}$.

To evaluate the overlapping area of these two squares we evaluate the area of trapezoid oabco; the overlapping area then equals four times the latter. The intersecting points of the rotated sides of the top square with the sides of the bottom one are labeled $a, b$, and $c$. Utilizing the coordinates of these points, the area of the trapezoid is the sum of the areas of two triangles $a b c$, and oac.

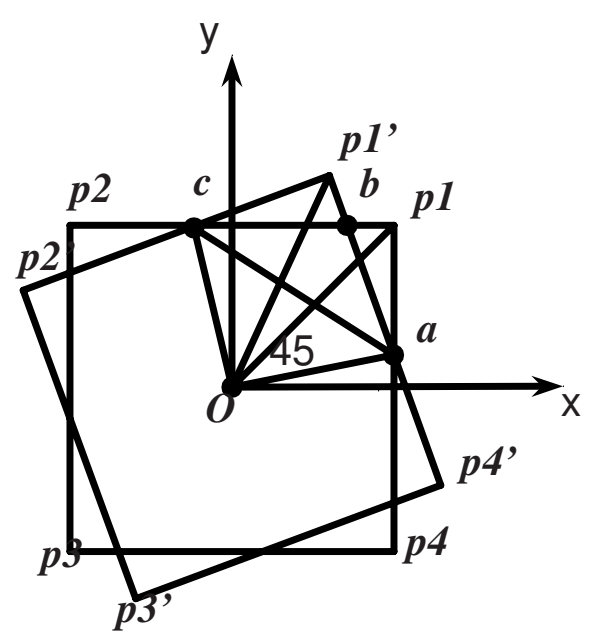

Fig. 1. Display of two rotated squares. The bottom square is fastened to the xy coordinate system, the top square is rotated counter clockwise by $\theta$ radian. 
To evaluate the coordinates of $a, b$, and $c$ we write the equations for the slanted lines, $P_{4}^{\prime} P_{1}^{\prime}$ and $P_{1}^{\prime} P_{2}^{\prime}$ and intersect them with the sides of the bottom square. Intersection of the former with the $P_{4} P_{1}$ and $P_{1} P_{2}$ gives the coordinates of $a$ and $b$ respectively. Similarly, the intersection of the latter with $P_{1} P_{2}$ yields the coordinates of $c$. Theses are: $a\left\{\frac{L}{2}, \frac{L}{2} \tan \left(\frac{\theta}{2}\right)\right\}, b\left\{\frac{L}{2} \frac{1-\tan \left(\frac{\theta}{2}\right)}{1+\tan \left(\frac{\theta}{2}\right)}, \frac{L}{2}\right\}$, and $c\{-$ $\left.\frac{L}{2} \tan \left(\frac{\theta}{2}\right), \frac{L}{2}\right\}$.

To evaluate the areas of the needed triangles, we convert the above coordinates into Mathematica code. The inserted 1's in the third position of the coordinates are for further calculations. $o=\{0,0,1\}, a\left[L_{-}, \theta_{-}\right]=\left\{\frac{L}{2}, \frac{L}{2} \tan \left[\frac{\theta}{2}\right], 1\right\}, b\left[L_{-}, \theta_{-}\right]=$ $\left\{\frac{L}{2} \frac{1-\tan \left[\frac{\theta}{2}\right]}{1+\tan \left[\frac{\theta}{2}\right]}, \frac{L}{2}, 1\right\}, c\left[L_{-}, \theta_{-}\right]=\left\{-\frac{L}{2} \tan \left[\frac{\theta}{2}\right], \frac{L}{2}, 1\right\}$. We define two auxiliary functions, $a b c\left[L_{-}, \theta_{-}\right]=\{a[L, \theta], b[L, \theta], c[L, \theta]\}, o a c\left[L_{-}, \theta_{-}\right]=\{o, a[L, \theta], c[L, \theta]\}$ The needed areas are, area $A B C\left[L_{-}, \theta_{-}\right]=\frac{1}{2} \operatorname{det}[a b c[L, \theta]]$,areaOAC $\left[L_{-}, \theta_{-}\right]=$ $\frac{1}{2} \operatorname{det}[$ oac $[L, \theta]]$,areaOABCO $\left[L_{-}, \theta_{-}\right]=$areaOAC $[L, \theta]+$ area $A B C[L, \theta]$.

We divide the overlapping area by the area of the square, $L^{2}$, and plot its normalized values as a function of the rotation angle $\theta$. Figure 2 shows the normalized area starts and ends at the same values. Its value after a $\frac{\pi}{4}$ radian turn drops to about $83 \%$ of the maximum value. The plot as one anticipates is symmetric about $\frac{\pi}{4}$.

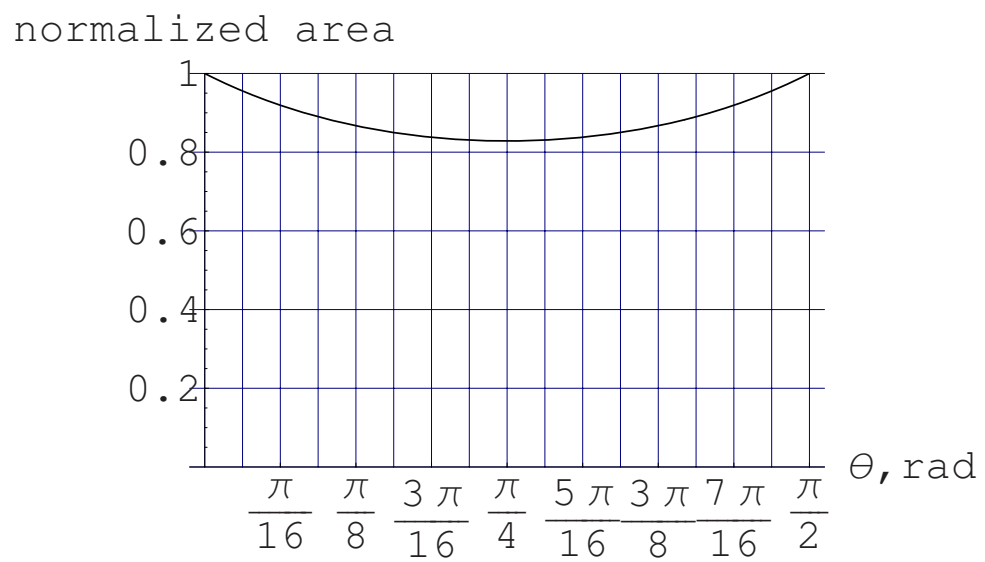

Fig. 2. The normalized values of the overlapping area of the squares as a function of the rotation angle $\theta$

\section{Modes of Mechanical Rotations}

In this section we extend the analysis of Section 2. Here, instead of viewing the rotation as being a discrete and purely geometrical concept, we view it as a kinematic process. We set the rotation angle $\theta=\omega t$; that is, we introduce the continuous time parameter $t$. For $\omega=\frac{2 \pi}{T}$ with the period $T=4 s$, we explore 
the uniform rotation. For an asymmetrical case, we consider a rotation with a constant angular acceleration. According to $\theta=\frac{1}{2} \alpha t^{2}$, to rotate the square by $\frac{\pi}{2}$ in one second yields $\alpha=\pi \frac{\mathrm{rad}}{\mathrm{s}^{2}}$. The corresponding normalized overlapping areas are displayed in Fig 3.

Show $[$ Graphics Array $[\{$ UniformRotation, AcceleratedRotation\}], DisplayFuncton $\rightarrow$ DisplayFunction].
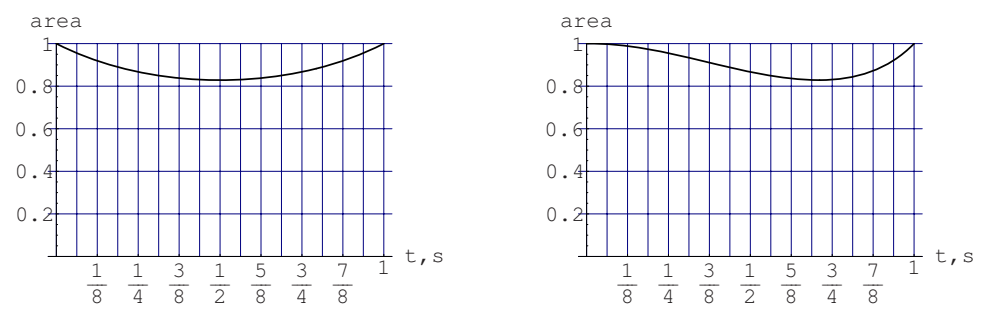

Fig. 3. The graphs are the normalized values of the overlapping areas for: a: a uniform rotation with $\omega=\frac{\pi}{2} \mathrm{rad} / \mathrm{s}$, (the left graph) and b: a uniform angular acceleration with $\alpha=\pi \frac{\mathrm{rad}}{\mathrm{s}^{2}}$ (the right graph)

\section{Electrical Networks}

Now we consider a RC series circuit. One such circuit driven by a DC power supply is shown in Figure 4. The circuit is composed of two loops. Throwing the DPDT (Double-Pole Double-Throw) switch to as position charges the capacitor, while setting the switch to a bs discharges the charged capacitor.

As we pointed out in the Introduction, in this section we view the overlapping squares as being two parallel metallic plates that are separated by a gap forming

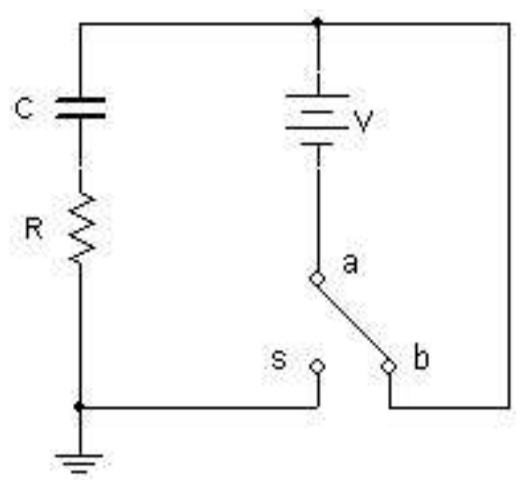

Fig. 4. The schematics of a DC driven RC circuit. Throwing the DPDT switch onto as charges the capacitor, while throwing the switch onto bs discharges the charged capacitor. 
a parallel-plate capacitor. Since the capacitor of a parallel-plate capacitor is in proportion to the overlapping area of the plates, the continuous rotation of the plates makes the capacitor time-dependent. It is the objective of this section to analyze the characteristic responses of one such time-dependent capacitor in the charging and discharging processes.

\section{Characteristics of Charging and Discharging a DC Driven RC Circuit with Time-Dependent Uniformly Rotating Plates}

For the charging process we apply Kirchhoff circuit law [2, this gives

$$
\frac{d Q}{d t}+\frac{1}{\tau} \frac{A_{0}}{A(t)} Q(t)-\frac{1}{\tau}=0,
$$

For the sake of convenience, we assume $V C_{0}=1$, where $C_{0}$ is the capacitance of the parallel-plate with the plates completely overlapped, $Q(t)$ and $A(t)$ are the capacitor's charge and the overlapping area at time $t$, respectively; $A_{0}$ is the area of one of the squares; and $\tau=R C_{0}$ is the time-constant of the circuit. For a constant capacitor $A(t) \equiv A_{0}$, and eq(1) yields the standard solution $Q(t)=1-e^{-\frac{t}{\tau}}$. In this equation the maximum charge is normalized to unity.

For the rotating plates, however, eq(1) does not have an analytic solution. We apply Mathematica NDSolve along with an appropriate initial condition and solve the equation numerically - this yields $Q(t)$. We graphically compare its characteristics vs. the characteristics of an equivalent RC circuit, see Fig 5.

Similarly, we analyze the characteristics of the discharging process. Equation (1) for the corresponding discharging process is $\frac{d Q(t)}{d t}+\frac{1}{\tau} \frac{A_{0}}{A(t)} Q(t)=0$. This equation for a constant capacitor, $A_{0}=A(t)$, yields $\frac{d Q(t)}{d t}+\frac{1}{\tau} Q(t)=0$, and gives $Q(t)=e^{-\frac{t}{\tau}}$. For the rotating capacitor, however, its solution is $Q(t)=$ $e^{-\frac{A_{0}}{\tau} \int_{0}^{t} \frac{1}{A(\xi)} d \xi}$. To solve the latter we apply Mathematica NIntegrate. This yields the needed values. The results are displayed in Fig 5.
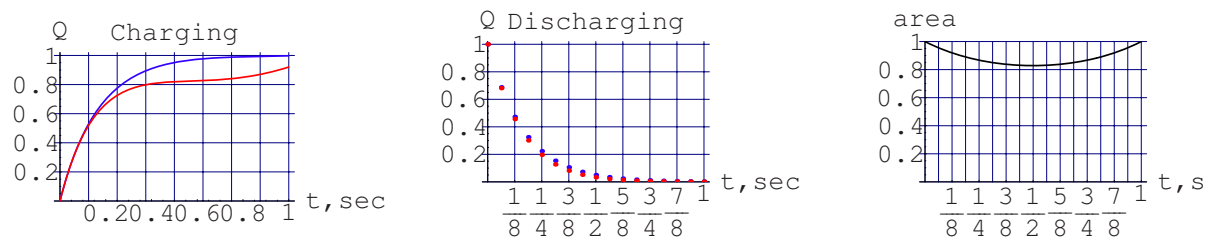

Fig. 5. Display of charging, discharging and the overlapping area of the uniformly rotating plates. For the first two graphs from left to right, the outer and the inner curves/dots represent the constant and time-dependent capacitors, respectively. The far right graph is borrowed from Fig 3.

It is interesting to note that the charging and discharging circuits respond differently to the time-varying capacitors; the impact of the time-dependent 
capacitor is more pronounced for the former. Moreover, for the chosen timeconstant $\tau=\frac{1}{6} \mathrm{~s}$, although the constant capacitor reaches its plateau within one second, it appears the variable capacitor requires a longer time span.

\section{Characteristics of Charging and Discharging DC Driven RC Circuit with Time-Dependent Accelerated Rotating Plates}

One may comfortably also apply the analysis of Section IIa to generate the characteristic curves associated with the uniformly accelerated rotating plates. The Mathematica codes may easily be modified to yield the needed information. The codes along with the associated graphic outputs are
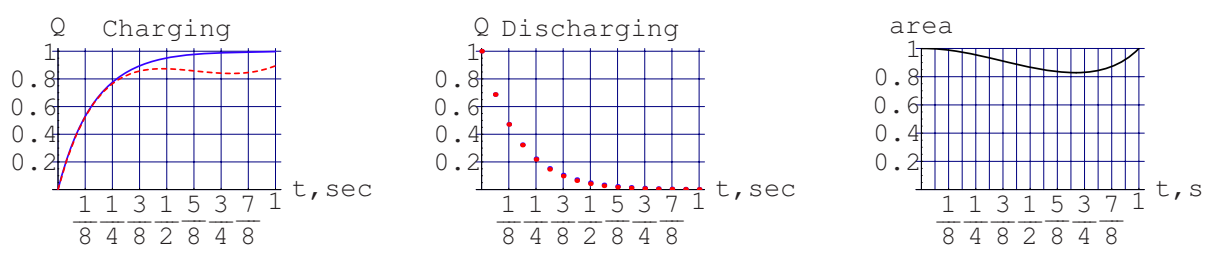

Fig. 6. Display of charging, discharging and the overlapping area of the uniformly accelerated rotating plates. The graph codes are the same as Fig 5. The far right graph is borrowed from Fig 3.

To form an opinion about the characteristics of the charging curve for the variable capacitor, one needs to view it together with the far right graph. The rotating plates in this case are accelerated, illustrating that for identical time intervals, the overlapping area at the beginning is greater than the overlapping area at the end of the interval. The effects of the asymmetrical rotation are most clearly visible at the tail of the curve. Similar to the uniform rotation (see the second plot of Fig 5) the impact of the non-uniform rotation for the discharge circuit is negligible.

\section{Characteristics of Charging and Discharging an AC Driven RC Circuit with Time-Dependent Capacitor}

In this section we analyze the charging and the discharging characteristics of an $\mathrm{RC}$ series circuit driven with an AC source. Schematically speaking, this implies in Fig 4 we replace the DC power supply with an AC source. For this circuit, Kirchhoff's law yields

$$
\frac{d Q}{d t}+\frac{1}{\tau} \frac{A_{0}}{A(t)} Q(t)-\frac{1}{\tau} \sin (2 \pi f t)=0,
$$


In this equation $f$ is the frequency of the signal and the voltage amplitude is set to one volt.

Equation (1) is a non-trivial, non-linear differential equation. To solve the eq(2), we apply NDSolve along with the corresponding initial condition. The response of the circuit is compared to the equivalent circuit with a constant capacitor.

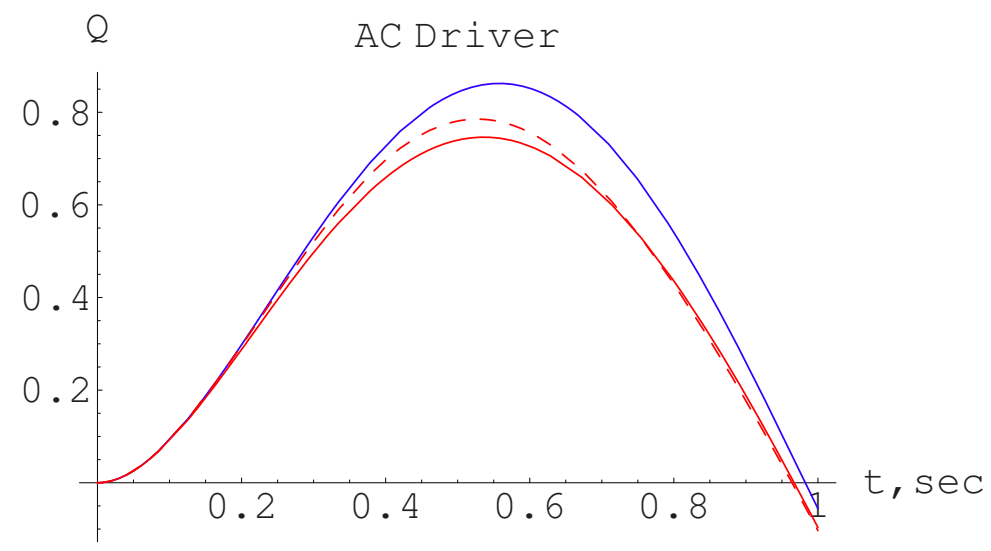

Fig. 7. Plot of the charge vs. time. The outer, inner and the dashed curves are the capacitor's charge for the constant and the time-dependent capacitors for uniform and accelerated rotations, respectively.

Utilizing the Mathematica code, one may analyze the frequency sensitivity of the circuit. As the result of one such analysis, we observe that the differences between these characteristics are pronounced, provided the frequencies are set to less than $1 \mathrm{hz}$.

\section{Conclusions}

As indicated in the Introduction, the authors have proposed a unique research project that has brought together three different subject areas: Geometry, Mechanics, and Electrical Network. Mathematica, with its flexible and easy to use intricacies, is chosen as the ideal tool to analyze the project and address the "what-if" scenarios. As pointed out in the text, some of the derived results are intuitively just. And for the hard to predict cases, we applied Mathematica to analyze the problem and to form an opinion. As an open-ended question and research oriented project, one may attempt to modify the presented analysis along with the accompanied codes to investigate the response of parallel $\mathrm{RC}$ circuits. It would also be complimentary to our theoretical analysis to manufacture a rotating capacitor to supplement the experimental data. 


\section{References}

1. S. Wolfram, The Mathematica Book, 5th Ed., Cambridge University Publication, 2003.

2. D. Halliday, R. Resnick, and J. Walker, Fundamentals of Physics, 7th Ed, New York: John Wiley and Sons, 2005. 